\title{
Functional Analysis Diagram of a Flux Modulated Magnetic Gear
}

\author{
Gheorghe Galmadi ${ }^{1, *}$ and Oana Dodun ${ }^{1}$ \\ ${ }^{1}$ Gheorghe Asachi Technical University of Iaşi, Department of Machine Manufacturing Technology, \\ 59A, Dimitrie Mangeron Bldv., Iaşi, 700050, România
}

\begin{abstract}
The reliability of a system is generally done by a functional analysis (FA), whose mission is to provide a deep understanding of the object to be designed, to discover all the valuable solutions, modes, and configurations, and finally to choose the best options for the system's design. Functional analysis is then matched with a dysfunctional analysis, such as Failure Modes and Effects Analysis, to foresee and take measures to avoid the possible dysfunctionalities of the system and to determine the most suitable model. In this paper, the functional analysis diagram (FAD) is used in the conceptual phase of the design process of a Flux Modulated Magnetic Gear.
\end{abstract}

\section{Introduction}

Magnetic Gear (MG) are used to transmit torque without mechanical contact between two shafts and resembles the traditional mechanical gear in geometry and function. The magnetic coupling uses a magnetic field instead of a physical-mechanical contact. The MG main feature consists of no-wear of the shaft seals or gear teeth surfaces, which means enhanced reliability of the device. More of that, the magnetic transmission does not require lubrication and has less noise in operation, the transmission has no local heating, no friction losses or risk of breaking the elements.

Mechanical gears have many industrial applications for power transmission. Even though their use is important, they have some inherent problems such as contact, overheat, friction, noise, and wear, which are often damaged in an over-torque situation and require periodic lubrication and maintenance. Magnetic gears can be solutions for these mechanical problems. Therefore, in recent years, magnetic gears (MGs) are increasingly receiving attention from both research institutions and industry; many authors have proposed several new magnetic gears to overcome these drawbacks. MGs offer lighter weight, reduced maintenance, high reliability, no wear and tear, contact-free operation, no noise and, higher efficiency.

However, magnetic gears have not attracted much attention since their initial development because of the low torque capability caused by the poor utilization of the permanent magnets [1]. In the past decade, a more efficient topology for magnetic gears was proposed in $[2,3]$. By adopting a coaxial arrangement of two permanent magnets

\footnotetext{
*Corresponding author: oanad@tcm.tuiasi.ro
} 
rings, all of the permanent magnets could contribute to the torque transmission simultaneously; hence a torque density exceeding $100 \mathrm{kN} \cdot \mathrm{m} / \mathrm{m}^{3}$ could be achieved [2].

This paper presents the concentric Magnetic Gears with a flux modulator, which can compete against certain mechanical constructions. The performances of different topologies and classification for the flux modulated magnetic gears available today are presented. The functional analysis methodology is used in order to achieve the necessary parameters of a certain magnetic gear for a certain purpose.

\section{Classification, characteristics, and performances of a concentric magnetic gear}

This section is dedicated to describing the most important concentric magnetic gears with flux modulator topologies that are available nowadays and present the best characteristics, have a buildable construction design, and were experimentally tested. Although there are

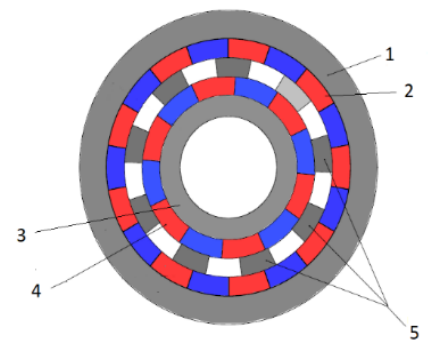

Fig. 1. Constructive scheme of a coaxial magnetic transmission with flux modulator:

1,2 - magnetic core and radial PM of the stator;

3,4 - Magnetic core and PM of the high-speed rotor; 5 - ferromagnetic pole-pieces of the low-speed rotor. many available topologies for which were obtained good characteristics after simulations, they do not have a practical design and applications because of difficulty or even impossibility to build them experimentally. These types of MG are much simpler to realize, considering the mechanical construction. Various aspects of this type of gear have been investigated.

Based on the orientation of magnetic flux, concentric MGs can also be divided into three categories namely:

- radial flux magnetic gear or coaxial magnetic gear (CMG) $[2,3]$;

- $\quad$ disc type or axial flux magnetic gear [4];

- and linear or transverse flux magnetic gear $[5,6]$.

Permanent magnets (PM) play an essential role in MG construction. Earlier MG designs suffered from poor torque producing capabilities of the available PMs at that time. With the realization of high-performance rare-earth magnets in the 1980s, there was a rapid positive shift in research and development in MG technology. Different types of MGs, which employ permanent magnets, can be divided into two big categories availability:

- MG with Non-Rare earth magnets: with Aluminium-Nichel_Cobalt (AlNiCo) permanent magnets or with Ceramic (Ferrite) PMs

- MG with Rare earth magnets: with Smaaium-Cobalt (SmCo) PMs or Neodymium-IronBoron (NdFeB) PMs

NdFeB PMs possess high magnetic strength but have low mechanical strength [7]. Due to its high magnetic strength, NdFeB was extensively used in MG research despite its high price.

The basic operation principle of a concentric MG is that the ferromagnetic pole-pieces interposed between the inner and outer PM rotors modulate the magnetic field such that each rotor "sees" a working space harmonic corresponding to its number of poles (Figure 1). The flux modulator is the key to the operation of concentric type MGs. It operates as either a lowspeed rotor (high torque) or the mechanical earth of an $\mathrm{MG}$.

In the absence of an intermediate ferromagnetic wheel, magnetic systems of external and internal magnetic rotors create magnetic fields with a different number of pairs of poles, and stable power transfer between them is impossible. When an intermediate magnetic rotor (modulator) is installed between the external and internal magnetic rotors, the magnetic field 
created by the external magnetic rotor will change its harmonic composition due to the presence of soft magnetic material segments in the modulator.

Different researchers have realized several prototype fluxmodulated gears until now. Table 1 lists some examples, with their outer diameter (Do), gear ratio (Gr), and active volume torque density (T/VA) reported. Although the majority of experiments could not exceed $100 \mathrm{kN} \cdot \mathrm{m} / \mathrm{m}^{3}$, in recent years, a few high torque magnetic gears have been experimentally obtained.
Table 1. MG performances from prototypes elaborated by different researchers.

\begin{tabular}{|l|c|c|c|}
\hline \multicolumn{1}{|c|}{ Authors } & $\begin{array}{c}\text { Outer } \\
\text { Diameter } \\
\mathrm{D}_{0}[\mathrm{~mm}]\end{array}$ & $\begin{array}{c}\text { Gear } \\
\text { ratio } \\
\mathrm{G}_{\mathrm{r}}\end{array}$ & $\begin{array}{c}\text { Torque } \\
\text { density } \\
\mathrm{T} / \mathrm{V}_{\mathrm{A}} \\
{\left[\mathrm{kNm} / \mathrm{m}^{3}\right]}\end{array}$ \\
\hline Atallah [2] & 140 & 5,5 & 72 \\
\hline Molokanov et al. [8] & 232 & 11.5 & 44.4 \\
\hline Rasmussen et al. [1] & 120 & 5.75 & 92 \\
\hline Frank and Toliyat [9] & 120 & 5.5 & 42 \\
\hline Valiente-Blanco [10] & $\sim 50$ & 10 & 95 \\
\hline Uppalapati et al. [11] & 112 & 4.25 & 33 \\
\hline Gerber and Wang [12] & 150 & 10.5 & 62 \\
\hline Fukuoka et al. [13] & 156 & 10.3 & 49 \\
\hline Shah et al. [14] & 120 & 5.5 & 56 \\
\hline Bird et al. [15] & 220 & 4.25 & 239 \\
\hline
\end{tabular}

\section{Functional Analysis of a concentric magnetic gear}

Functional Analysis (FA) is a fundamental tool of the design process to discover new meaningful concepts, to define their structure, and to have a deep understanding of the researched object [16]. FA is used in engineering to define the functional requirements of the new product, to map its functions to physical components by listing all the necessary components, and, where is needed, by eliminating the unnecessary ones, a method that will help designers to understand the relationship between product's components.

Functional analysis is particularly useful during the conceptual design phase, as it provides a clear understanding of functions related to energy, materials and signals and can help choose the best option among a wide range of feasible solutions discovered in the process of exploring ideas that may offer significant advantages.

The method is also used to define material limits, the operations, and functions performed by the system and its configurations. A practical way of using FA is by visually representing the decomposed system, braking its functions down into sub-functions, providing a format, and a specified set of rules to perform FA of all functions of the system [17].

Functional analysis is performed in two stages [18]: external functional analysis which shows the relations between the system and its external environment and internal functional analysis which perform breakdown of the system into elements and describes the functions of the system but also the relationships between the components and therefore the physical interactions between the different entities of the system.

Although FA is relatively easy to use and its application is similar to any system, its steps are adaptable and defined by the various aspects of the analyzed object. A common set of steeps are described in [19]: identification of system states and modes; development of the functional tree of all functions the system; identification of flow relationships across functions; identification of function interfaces; allocation of requirements to functions; identification of function time synchronization requirements.

The researches on functional analysis led to development of such representation methods as Function Tree, Data Flow Diagram, Function Structure and FAST Diagram [20].

A form-dependent method for functional representation is the function analysis diagram (FAD) which uses blocks to represent device or system structure and arrows with labels to represent functional relations between components. FAD originated in Invention Machine Goldfire software based upon TRIZ methodology [17] as a tool which visually represents the 
decomposed system, providing the space and a specified set of simple rules to perform a functional analysis of all functions of a system at any level [20].

FAD orders the functions in a logical order, it contributes to the clarification of the functional state of the product and it allows to order the functions identified by the designer or the analyzer, to have a good knowledge of the product or system studied thus become aware of the relative importance of elements or structures in relation to the functions they perform which results in synchronizations between independent functions.

The methodology is used to examine external and internal functions concerning a potential magnetic transmission of an electric car. External Functional Analysis displays the relation between the transmission and the systems to which

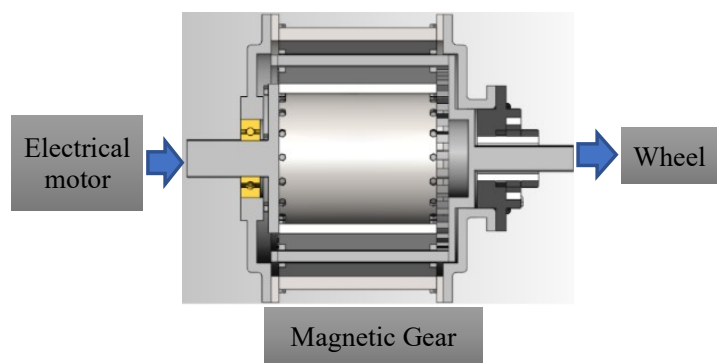

Fig. 2. Magnetic Gear of an electric car and its relation with connected systems. it is connected, in our case, the electrical engine and the wheel, and the internal FA lists all the functions of the magnetic transmission (Figure 2).

Usually, Functional Analyses Diagrams (FAD) are developed for large systems that incorporate many subsystems, but here, it is analyzed a smaller assembly, such as a magnetic gear. The diagram is developed using a hierarchical structure.

The relationship between two sub-systems or system items can be represented in the diagram as relationship between: a) system function and the equipment implementing it; b) system function and the system states requiring it; c) internal interfaces between system functions; d) system external interfaces; e) system function and performance requirements [19]. The first step starts with the analysis of primary gearbox functions and their interactions with the peripherical systems, as shown in Figure 3.

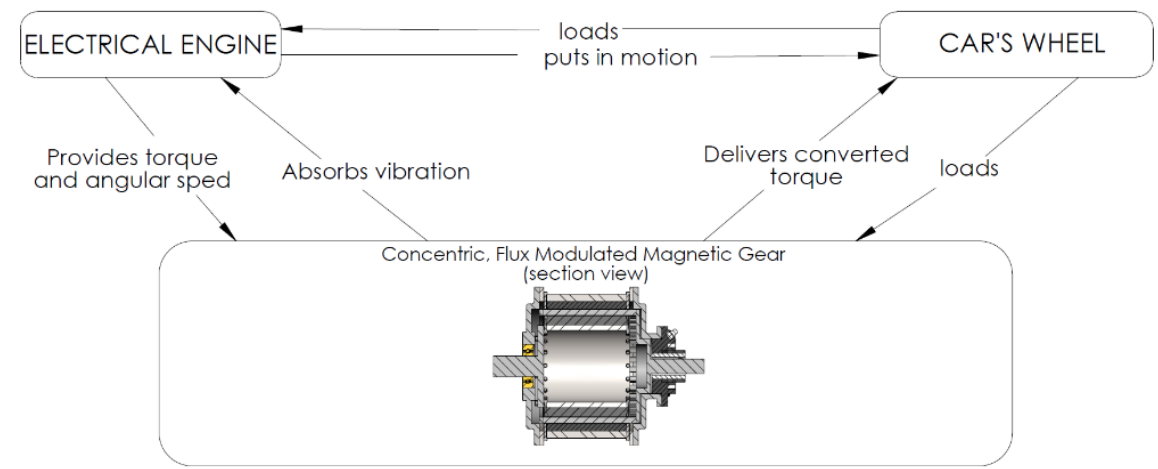

Fig. 3. Top-level functions of MG of an electric vehicle and its interactions with the electrical engine and wheel.

The product's top-level functional diagram contains its primary product functions, in our case, the primary function of the MG is to take over the torque from the engine and to deliver it to the car's wheel at a necessary angular speed and torque. In the top-level FAD model, there can also be found additional functions such as vibration absorption and noise cancelation in magnetic gear due to the nature of magnetic interaction between the rotors.

The second or middle level of the FAD examines the subassemblies of the MG and the functions that link them. Every arrow represents a function a subassembly exerts upon another and the way the entire assembly works. This type of representation will be very useful 
when Failure Modes and Effects Analysis are done as every function could be analyzed to evaluate the probability of failure.

All the functions represented at the level of the FAD sub-assembly work together to ensure the execution of the functions of the assembly. The representation from Figure 4 provides a general understanding of how the Magnetic Gear is assembled and how it works.

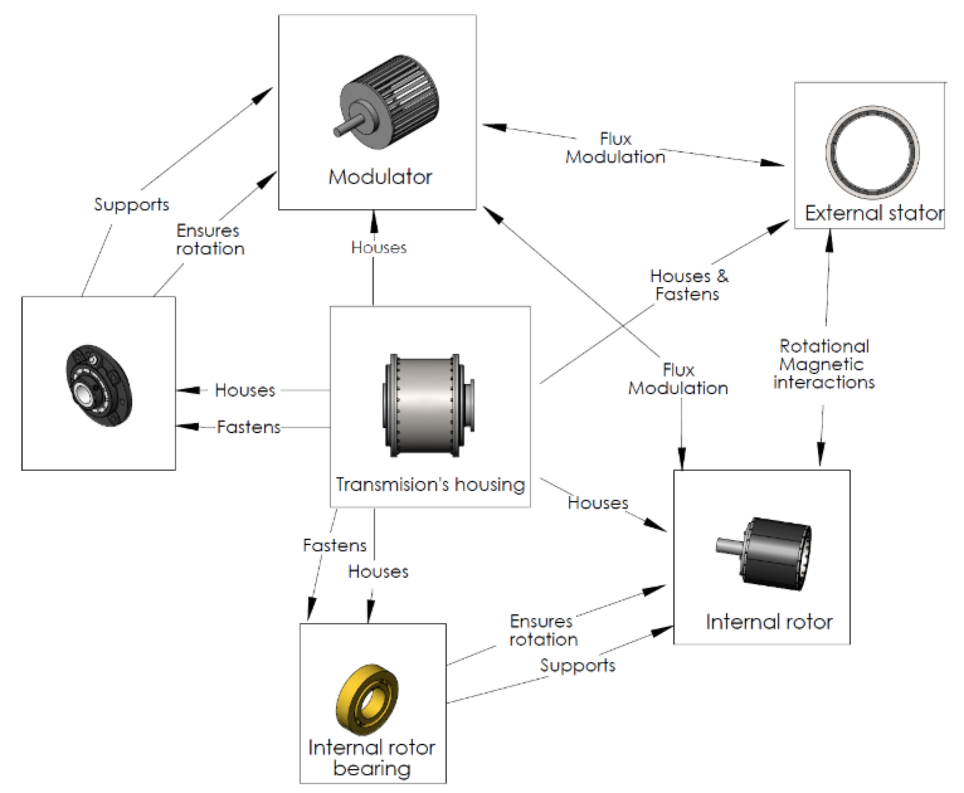

Fig. 4. Sub-assembly level of MG diagram and the functional interaction between them.

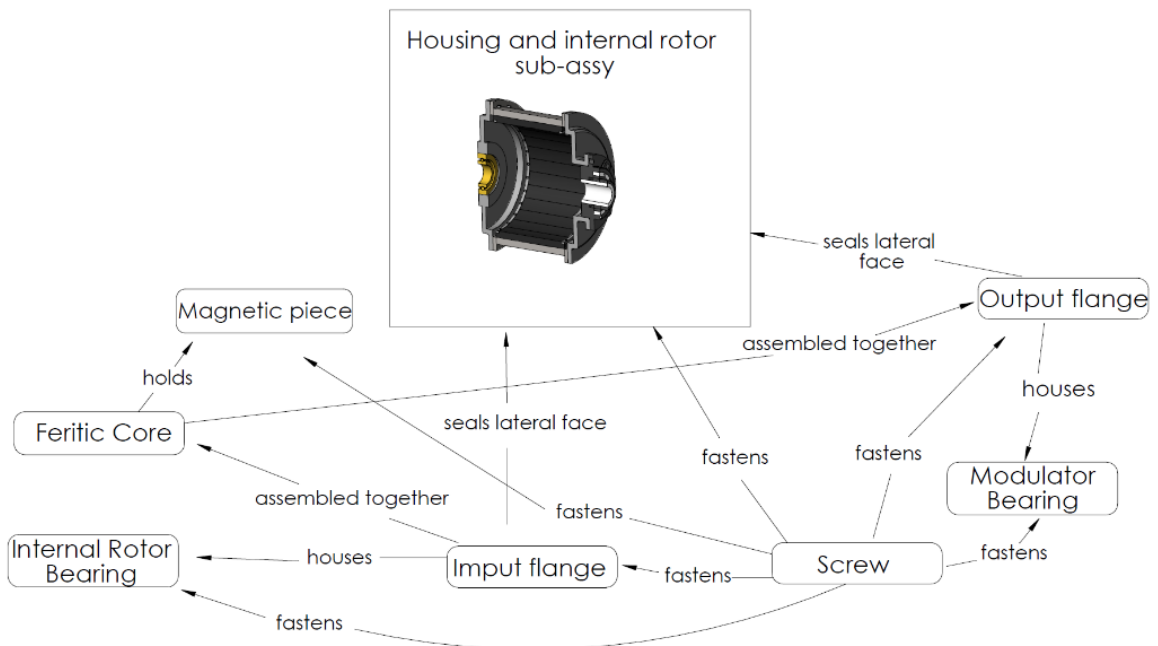

Fig. 5. Functional Diagram.

The final step is the development of the functional analyses diagram represents and explains the function of every component and its utilization in the assembly. FAD could be useful for analyzing the necessity of a certain component and a possible replacement with another one that does the same function in a better fashion, or if the function is not necessary, the component could be eliminated to simplify the assembly design (Figure 5). 


\section{Conclusion}

In this paper, an overview of concentric magnetic gear with a flux modulator has been presented. Although the emphasis was on radial flux concentric MG, the other two types require further analysis because of the advantages they present. The growing capabilities of this type of MG in recent years due to optimized topologies and better use of PM properties make possible their serial manufacturing and practical application in diverse industries. For this reason, a Functional Analysis related to the design process of MG's has been done to facilitate the Design Process of the Magnetic Gear. The method has shown capability for optimization of the magnetic gear design and displays a possible utilization in a interdisciplinary environment due to its quality to support high levels of understanding trough functional decomposition. The future works will use the functional analyses diagrams of this type of MG to utilize it in the process of design failure mode and effect analysis (DFMEA) of such a system.

\section{References}

1. P.O. Rasmussen, T.O. Andersen, F.T. Jorgensen, O. Nielsen, IEEE Trans. Ind. Appl. 41, 764-770 (2005)

2. K. Atallah, D. Howe, IEEE Trans. Magn. 37, 2844-2846 (2001)

3. K. Atallah, S. Calverley, D. Howe, IEE Proc. Electr. Power Appl. 151, 135-143 (2004)

4. S. Mezani, K. Atallah, D. Howe, J.of Appl. Phys. 99(8):08R303-08R303-3 (2006)

5. K. Atallah, D. Howe, J. of Appl. Phys., 97(10):10N516-10N516-3 (2005)

6. N. Niguchi, K. Hirata, M. Muramatsu, Y. Hayakawa, Proceedings of the 14th Biennial IEEE Conference on Electromagnetic Field Computation (2010)

7. M. Todorova, V. Mateev, I. Marinova, Proceedings of the 19th International Symposium on Electrical Apparatus and Technologies SIELA, 1-4 (2016)

8. O. Molokanov, P. Dergachev, V. Kiruhin, P. Kurbatov, Proceedings of 18th International Symposium on Electrical Apparatus and Technologies SIELA, (2014)

9. N.W. Frank, H.A. Toliyat, IEEE Energy Conv. Congress and Exposition ECCE, 29772984 (2010)

10. I. Valiente-Blanco, J.L. Perez-Diaz, E. Diez-Jimenez, Efren, in 44th Aerospace Mechanisms Symposium, 255-262 (2018)

11. K. Uppalapati, W. Bomela, J. Bird, M. Calvin and J. Wright, IEEE Energy Conv. Congress \& Expo. ECCE, 2178-2184 (2013)

12. S. Gerber and R.-J. Wang, IEEE Int. Conf. on Ind. Tech. ICIT, 319-324 (2013)

13. M. Fukuoka, K. Nakamura and O. Ichinokura, Int. Conf. Electr. Machines \& Sys. (2012)

14. L. Shah, A. Cruden, B. Williams, Int. Conf. on Power Electron. \& Drive Sys. PEDS, 989-993 (2007)

15. J. Bird, Proceedings of IEEE Energy Conversion Congress and Exposition (ECCE), 1422 (2014)

16. N. Viola, S. Corpino, M. Fioriti, F. Stesina, Functional Analysis in Systems Engineering: Methodology and Applications, (InTech, 2012)

17. M. Atherton, P. Jiang, D. Harrison, A. Malizia, Res. Eng. Des. 29(2), 245-262 (2018)

18. J. Ben Salem, M.N. Lakhoua, L. Amraoui, Int. Conf. on Electrical Sciences and Technologies in Maghreb (CISTEM), IEEE Xplore (2014)

19. T. Pinna, D.N. Dongiovanni, F. Iannone, Fusion Eng. Des. 109-111 (Part A), 795-800 (2016)

20. I. Michalakoudis, P.R. Childs, M. Aurisicchio, N. Pollpeter, N. Sambell, ASME 2014, V011T14A011 (2014). 